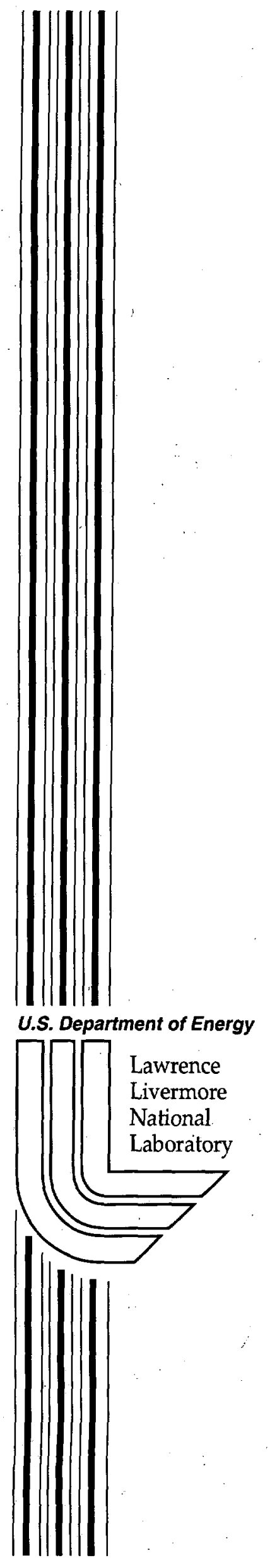

\title{
Edge-Plasma Properties in Liquid-Wall Environments
}

\author{
T.D. Rognlien, M.E. Rensink, J.N. Brooks
}

This article was submitted tò $8^{\text {th }}$ International Workshop on Theory in Fusion Devices, Espoo, Finland, September 10-12, 2001

\section{September 7, 2001}




\section{DISCLAIMER}

This document was prepared as an account of work sponsored by an agency of the United States Government. Neither the United States Government nor the University of California nor any of their employees, makes any warranty, express or implied, or assumes any legal liability or responsibility for the accuracy, completeness, or usefulness of any information, apparatus, product, or process disclosed, or represents that its use would not infringe privately owned rights. Reference herein to any specific commercial product, process, or service by trade name, trademark, manufacturer, or otherwise, does not necessarily constitute or imply its endorsement, recommendation, or favoring by the United States Government or the University of California. The views and opinions of authors expressed herein do not necessarily state or reflect those of the United States Government or the University of California, and shall not be used for advertising or product endorsement purposes.

This is a preprint of a paper intended for publication in a journal or proceedings. Since changes may be made before publication, this preprint is made available with the understanding that it will not be cited or reproduced without the permission of the author.

This report has been reproduced directly from the best available copy.

Available electronically at http://www.doe.gov/bridge

Available for a processing fee to U.S. Department of Energy and its contractors in paper from

U.S. Department of Energy

Office of Scientific and Technical Information

P.O. Box 62

Oak Ridge, TN 37831-0062

Telephone: (865) 576-8401

Facsimile: (865) 576-5728

E-mail: reports@adonis.osti.gov

Available for the sale to the public from

U.S. Department of Commerce

National Technical Information Service 5285 Port Royal Road Springfield, VA 22161

Telephone: (800) 553-6847

Facsimile: (703) 605-6900

E-mail: orders@ntis.fedworld.gov

Online ordering: http://www.ntis.gov/ordering.htm

OR

Lawrence Livermore National Laboratory Technical Information Department's Digital Library http://www.llnl.gov/tid/Library.html 
Contrib. Plasma Phys. vol (year) num, $\mathrm{p}-\mathrm{p}$

\title{
Edge-Plasma Properties in Liquid-Wall Environments
}

\author{
T.D. ROGNLIEN ${ }^{a}$, M.E. RENSINK ${ }^{a}$ AND J.N. BROOKS ${ }^{b}$ \\ ${ }^{a}$ Lawrence Livermore National Laboratory, Livermore, CA 94551, USA \\ ${ }^{\mathrm{b}}$ Argonne National Laboratory, Argonne, IL 60439, USA \\ e-mail: trognlien@llnl.gov
}

\begin{abstract}
Flowing liquid walls have been proposed as the first wall and/or divertor plates for magnetic fusion energy devices because they may solve a number of technological problems for fusion power plants. A key question for their successful use is the edge-plasma shielding of wall-vapor impurities from the core plasma. A self-consistent analysis of the combined hydrogen/impurity edge plasmas for distributed wall impurity sources is performed using the two-dimension fluid transport code UEDGE for tokamak parameters. Three regimes of edge-plasma response are identified. Comparisons are made between previous results for lithium (from $\mathrm{Li}$ or SnLi walls) and fluorine (from the molten salt Flibe walls), and new results for $\mathrm{Sn}$ (from $\mathrm{Sn}$ walls). Owing to its lower vapor pressure, $\mathrm{Sn}$ is found to have the lowest impact on the edge and core plasma. For the Sn wall, the effects of toroidal versus slab geometries are studied, as well as the influence of spatial variations in evaporation fluxes.
\end{abstract}

\section{Introduction}

The edge-plasma region of a fusion device plays the important roles of distributing the escaping charged-particle energy from the hot core plasma to the surrounding material surfaces, helping determine the core-edge temperature and density values, and shielding the core from wall impurities. Extensive theoretical and experimental progress has been made in understanding the edge plasma properties for tokamaks with divertor configurations utilizing solid divertors and walls, e.g., [1]. While there have been previous work on using liquids, recently there has been renewed interest in for using flowing liquid walls and divertors to increase the heat-load capabilities of surfaces,- negate the material erosion problem, and reduce neutron activation of stationary walls and supporting structures $[2,3,4]$; these references discuss work on issues such as liquid hydrodynamic stability, wall coverage, and thickness possible both low and high electrical-conductivity flowing liquids in fusion devices with strong magnetic fields. Here we analyze the impact of the evaporating vapor from such a wall on the edge and core plasmas.

The use of liquids results in a potentially large impurity source owing to the strongly temperature dependent evaporating rate. We present results on the modifications to the edge-plasma from the resulting distributed wall-impurity source, and determine the range of allowable surface temperatures of the liquid based on core impurity limits. We have previously studied fluorine (F) vapor coming from the low-conductivity molten salt $\mathrm{Li}_{2} \mathrm{BeF}_{4}$ and lithium vapor coming from $\mathrm{Li}$ or $\mathrm{SnLi}$ walls in a simple slab model approximating a tokamak with a uniformly distributed impurity wall source [5]. This earlier paper also pointed out the substantial difference in core penetration expected between low- and high-recycling divertor operation.

The present paper identifies the edge-plasma characteristics for three regimes as the impurity source is increased, ranging from the trace impurity limit to the edge- 
collapse regimes. We extend the previous work in slab-tokamak geometry to include tin $(\mathrm{Sn})$, which because of its low vapor pressure, results in comparatively low core contamination despite its high nuclear charge, $Z=50$. Using the Sn case, we demonstrate the effect of toroidal tokamak geometry and of the more realistic spatial profiles for the wall evaporation rate. While we focus on impurities from the side-wall, analysis has also been done for the impurities from liquid divertors [6]. Owing to the larger heat and particle fluxes to the divertor plates compared to the side wall, the impurity vapor can often be ionized within the plasma sheath (electrostatic+magnetic) of the divertor surface, thereby greatly reduce its migration to the core region.

The plan of the paper is as follows: The geometry and physics models are discussed in Sec. 2. Three impurity-induced edge-plasma regimes are identified in Sec. 3, while Sec. 4 presents comparisons of $\mathrm{Sn}$ walls with previous Flibe (F) and lithium ( $\mathrm{Li}$ and $\mathrm{SnLi})$ results. Geometry and nonuniform vapor flux effects are illustrated in Sec. 5, and the conclusions are given in Sec. 6 .

\section{Geometry, physics models, and parameters}

Two related models for the tokamak geometry are used as shown in Fig. 1. The more complete model given on the left is based on the geometry obtained from the magnetic field, B, via an MHD equilibrium calculation (here, a slight variant of ARIES-RS [7] to yield a single-null divertor), and to the right is a slab approximation. We use the slab model to make comparison with results from Ref. [5], and then compare with the toroidal geometry in Sec. 5 .

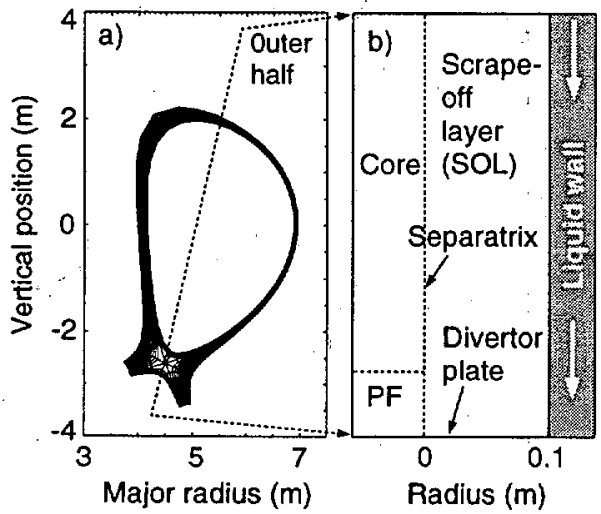

Fig. 1: Correspondence between a), full tokamak edge region, and b), slab model. Example a) is ARIES-RS, modified to a single-null divertor.
Two slab cases are considered with total poloidal lengths of $L_{p}=14.5 \mathrm{~m}$ and $7.25 \mathrm{~m}$; the former matches previous ITERlike calculations [5], while the half-sized case matches the outer half of ARIES as shown. For the slab cases, ratio of the poloidal to toroidal B-field is $B_{p} / B_{t}=0.17$, which is between the range for the toroidal case.

The UEDGE 2D transport code to calculate the spatial distribution of the hydrogen and the impurities [8]. Equations are solved for particle continuity and parallel momentum for each ion charge-state, where the parallel direction is that along $\mathbf{B}$. Ion inertia is neglected for impurities in the high-recycling cases only. The inertialess parallel electron momentum equation is used to determine the parallel electric field, $E_{\|}$, in terms of the electron pressure. Separate electron and ion temperature equations are used, with single summed equation for all ions and neutrals. Recycling of hydrogen ions at surfaces is set to 0.99 (high) or 0.25 (low), while there is no recycling of impurities. The hydrogen neutrals are described by a reduced Navier-Stokes model[9], and the impurity neutral density is calculated with a diffusive model (i.e., inertia and viscosity are neglected) with a single-component neutral temperature fixed at $1 \mathrm{eV}$. This temperature is about that expected from dissociation of Flibe molecules, whereas for $\mathrm{Li}$ or $\mathrm{Sn}$ it requires some plasma heating. However, results for $\mathrm{Li}$ using $0.1 \mathrm{eV}$ show very little difference in the maximum evaporative flux 


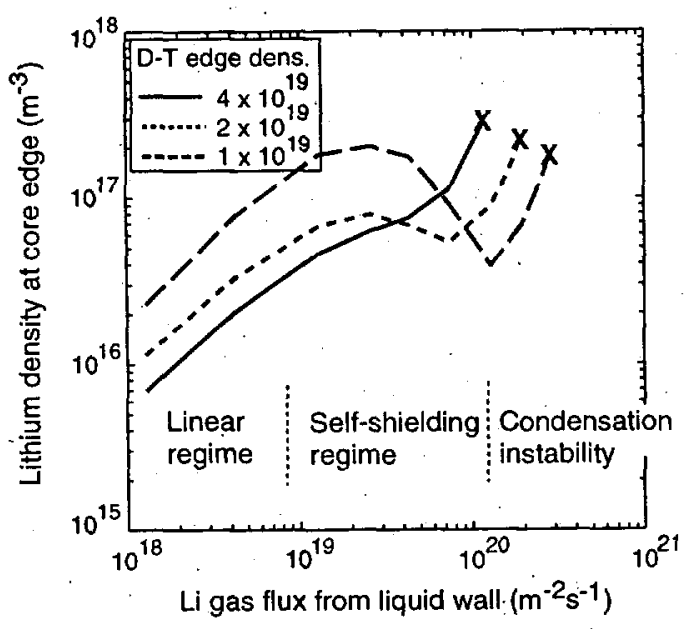

Fig. 2: Core-edge Li density for $14.5 \mathrm{~m}$ slab case with low-recycling hydrogen for various $n_{D T}$ showing the 3 regimes.

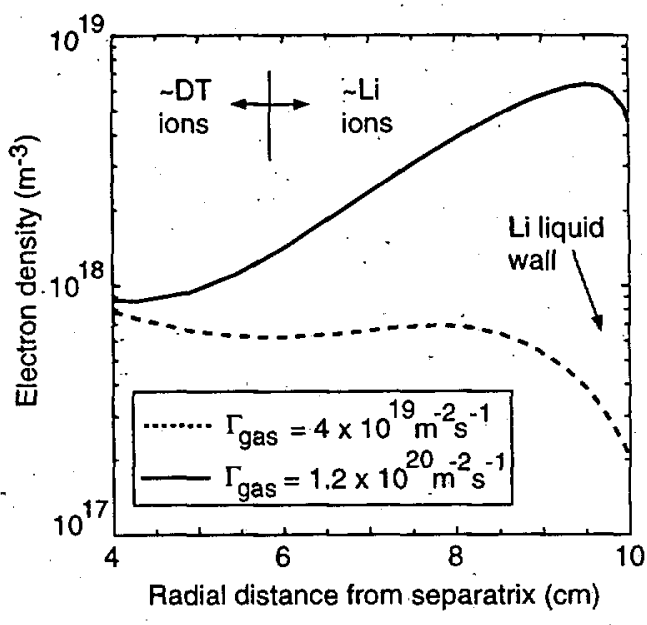

Fig. 3: Electron density, $n_{e}$, for two $\mathrm{Li}$ wall gas-fluxes with $n_{D T}=1 \times 10^{19} \mathrm{~m}^{-3}$ showing the build up of the Li shield.

allowed. The ionization and radiation rates for hydrogen and the impurities are taken from Refs. [10, 11], respectively.

The parallel transport is assumed to be classical [12] with flux-limits on the viscosity, thermal force, heat conductivity terms. Flux limits are also used for the hydrogen and impurity neutrals. The cross-field transport is assumed to be diffusive owing to plasmas turbulence, with typical values from a survey of present experimental results [1]. We use coefficients for density of $D=0.33 \mathrm{~m}^{2} / \mathrm{s}$, for electron and ion energy transport, $\chi_{e, i}=0.5 \mathrm{~m}^{2} / \mathrm{s}$; radial ion viscosity is also set to $0.5 \mathrm{~m}^{2} / \mathrm{s}$. In addition, these anomalous cross-field diffusion coefficients are not allowed to exceed the Bohm rate, $D_{B}=T_{e} / 16 e B$. The parallel energy flux of electrons and ions to the divertor plate is taken as $\delta_{e, i} n T_{e, i} c_{s}$, where $\delta_{e, i}=5$ and 2.5 , respectively, and $c_{s}=\left[\left(T_{e}+T_{i}\right) / m_{i}\right]^{1 / 2}$.

\section{Edge-plasma regimes induced by impurities}

We identified three edge-plasma regimes observed as the source strength of the impurities is increased. In addition, the hydrogen recycling at the divertor plate can play an important role in the impurity build-up in the scrape-off layer (SOL) as discussed in Ref. [5], and both recycling cases are considered in the next section.

The three impurity regimes are most clearly seem in a low-recycling case with Li. We use the slab model with $L_{p}=14.5 \mathrm{~m}$ and vary the uniform side-wall impurity gas fluxes for three hydrogen core-boundary densities, $n_{D T}$. The resulting Li density at the core boundary is shown in "Fig. 2. At low flux, the impurities don't change the hydrogenic edge plasmas, yielding the linear regime with impurity density directly proportional to the flux. For increased fluxes, a self-shielding regime causes the rate-ofrise of the core density to decrease and sometimes change sign. Here, a predominately Li plasma forms very close to the Li wall, as shown in Fig. 3 near the poloidal midplane; sufficient $n_{e}$ develops to ionize Li gas near the wall, so ions can be readily lost axially before they diffuse to the core. For higher hydrogen densities and/or higher recycling, the self-shielding regime becomes less effective. At the highest gas fluxes, the radiation loss becomes large, causing the width of the SOL to shrink. The resulting reduced 

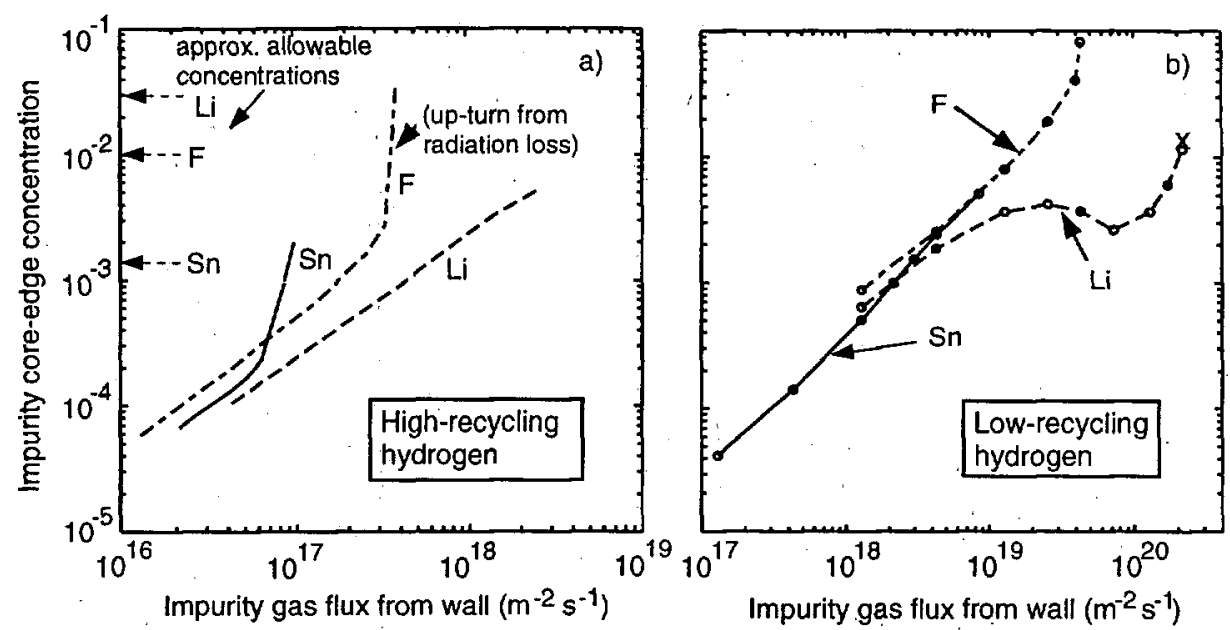

Fig. 4: Impurity concentration for slab model with $L_{p}=14.5 \mathrm{~m}$ versus uniform gas flux; a) is for high-recycling divertor with $n_{D T}=4 \times 10^{14} \mathrm{~m}^{-3}$, and b) has low-recycling with $n_{D T}=2 \times 10^{14} \mathrm{~m}^{-3}$.

parallel impurity loss (width $\times$ parallel-flux), leads to a radiation collapse of the SOL (a radiation/condensation instability).

\section{Comparison of Sn walls with other liquids}

Previous impurity-intrusion results [5] focused on $\mathrm{Li}$, SnLi, and Flibe liquids, for which $\mathrm{Li}$ and $\mathrm{F}$, are the most limiting for core contamination ( $\mathrm{SnLi}$ evaporates very little $\mathrm{Sn}$ ). Here we compare those results of core-boundary impurity concentrations versus gas flux with those for a pure Sn wall in Fig. 4. Both high-recycling $\left(R_{h}=0.99\right)$ and low-recycling $\left(R_{h}=0.25\right)$ are given. In the linear regime, the $\mathrm{Sn}$ concentration is very similar to $\mathrm{Li}$ and $\mathrm{F}$, but there an earlier up-turn from radiation collapse for Sn owing to its higher nuclear charge. The upper endpoints of the curves in Fig. 4 represent a thermal collapse of the divertor plasma, and steady-state solutions could not be obtained at higher fluxes. The impurity concentration limits for the different impurities shown in Fig. $4 \mathrm{a}$ are taken from a combination of core fuel dilution and radiation loss, and are only to be consider as approximate; these assume the impurity density (not concentration) is uniform within the core, and must be revised with another assumption.

In spite of the lower limit on $\mathrm{Sn}$, the allowable wall temperature can in fact be quite large, owing to its low vapor pressure. The vapor flux, $J$, from the wall can be given in terms of the liquid surface temperature, $T_{s}$ from vapor pressure data as $[3,5]$

$$
J=\frac{7.94 \times 10^{34}}{T_{s}^{1 / 2}} \exp \left(\frac{-3.81 \times 10^{4}}{T_{s}}\right),
$$

where $T_{s}$ is in ${ }^{\circ} \mathrm{K}$, and $J$ is in $\mathrm{m}^{-2} \mathrm{~s}^{-1}$. For comparison, at a given $T_{s}$, this flux is about a factor of $10^{-6}$ less than the Flibe flux [5], allowing the Sn wall to operate at much higher temperatures. Thus, from Fig. $4 a$, we obtain an allowable Sn temperature of $1000^{\circ} \mathrm{K}$ for high recycling, which is the expected recycling regime if $\mathrm{Sn}$ is also used for the divertor surfaces. 


\section{Toroidal geometry and nonuniform vapor flux}

A variety of factors can influence the maximum allowable impurity flux (and thus wall temperature), and here we discuss two: geometry and poloidal vapor profile. For the geometry, we keep the power flux across the core-boundary fixed to $1.4 \times 10^{5} \mathrm{~W} / \mathrm{m}^{2}$, but shorten the poloidal length from $14.5 \mathrm{~m}$ to $7.25 \mathrm{~m}$. There is also a small change in $n_{D T}$ from $4 \times 10^{19} \mathrm{~m}^{-3}$ to $5 \times 10^{19} \mathrm{~m}^{-3}$ for the $7.25 \mathrm{~m}$ case, but this has little effect. The results for Sn walls are shown in Fig. 5; the $14.5 \mathrm{~m}$ curve is the same as in Fig. 4 . The shorter slab has delays the thermal collapse of the divertor, giving about a factor of 2 larger allowable flux. The nominal concentration limit is assumed to be $\sim 10^{-3}$, and the endpoints of the curves denote the thermal collapse.

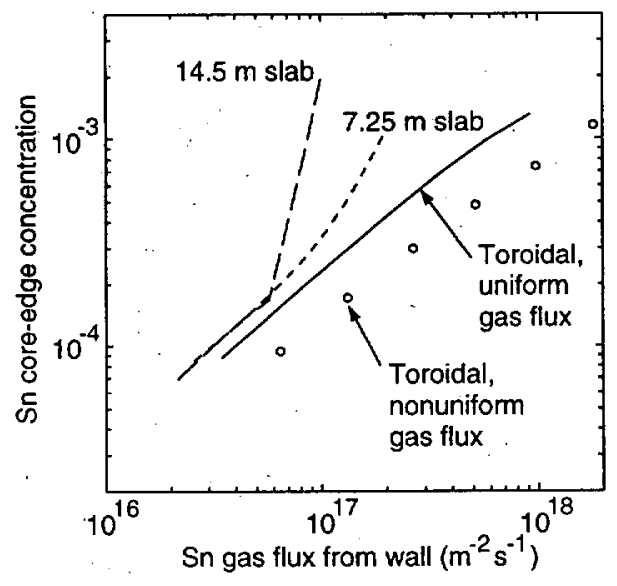

Fig. 5: Comparison of core impurity influx for 3 geometry variations. Points correspond to strongly nonuniform evaporation from wall heating model.

The next variation is to use the outerhalf of the modified ARIES-RS case shown in Fig. 1 with a poloidal length of $7.1 \mathrm{~m}$. Here, the ratio of $B_{p} / B_{t}$ depends on position, and correspondingly, so does the flux tube area. The results show that the linear stage is quite similar in the linear regime, but does not show the same rapid up-turn as the slab curves as the thermal collapse endpoint is approached. This results in an increase in the allowable $\mathrm{Sn}$ flux of $\sim 3.5$ over the corresponding $7.25 \mathrm{~m}$ slab case.

Finally, we consider a more realistic walltemperature, and thus, evaporative flux, profile. The liquid wall is heated by Bremsstrahlung and line-radiation; the surface temperature $T_{s 0}$ as it enters the chamber at the top of Fig. 1, and heats as it flows to the exit near the divertor. Considering the radiation to be deposited uniformly at the wall surface, and heat flow into the liquid to be by conduction, gives a temperature profile of $T_{s}=T_{s 0}+\Delta T\left(x / L_{p}\right)^{1 / 2}$, where $x$ is the poloidal position measured from the inlet. A value of $\Delta T \approx 200^{\circ} \mathrm{K}$ results. Because of the strong $T_{s}$ dependence of $J$ in Eq. (1), the gas flux is strongly peaked toward the outlet. The points in Fig. 5 correspond to the toroidal case with $T_{s 0}$ varying between $860^{\circ} \mathrm{K}$ and $940^{\circ} \mathrm{K}$, with the abscissa now being the poloidally-averaged gas flux. Again, the upper-most point is very close to thermal collapse of the divertor plasma; now the effective temperature giving the same flux from Eq. (1) is $\sim 1100^{\circ} \mathrm{K}$, while $T_{s}$ ranges over $940-1140^{\circ} \mathrm{K}$.

\section{Conclusions}

An analysis has been presented for the impurity transport and edge-plasma modification in the edge region of tokamak configurations where the impurities arise from a distributed liquid-wall vapor. A principle result is the core-boundary impurity density as a function of the vapor flux, whose maximum allowable value then specifies the allowable liquid surface temperature.

Three edge-plasma regimes are identified as induced by the impurities: linear, self-shielding, and radiation-caused condensation or energy collapse. Each regimes is clearly seen for low-recycling Li divertors, whereas self-shielding is generally absent 
for the high-recycling regime. Tin vapor is shown to have lower allowable vapor-flux for core contamination compared to F (from Flibe) or $\mathrm{Li}$ (from $\mathrm{Li}$ or $\mathrm{SnLi}$ ), but Sn's much lower vapor pressure allows it to operate at much higher surface temperatures. Including more detailed geometry and evaporation profile models both increase the allowable vapor flux; for $\mathrm{Sn}$ in an ARIES-like configuration with a core-boundary energy flux of $1.4 \times 10^{5} \mathrm{MW} / \mathrm{m}^{2}$ yields an effective surface temperature limit of $1100^{\circ} \mathrm{K}$.

Although several parameter variations have been explored here, others need to be considered. Some changes to the anomalous cross-field diffusion coefficients have been made, with a fairly weak dependence found. The core impurity profiles should be studied. The impact of possible non-diffusive edge transport needs to be considered, as well as a more detailed treatment of long mean-free path effects in the low density, high temperature plasmas found for the low-recycling cases. Nevertheless, the present results indicate the a $\mathrm{Sn}$ wall looks promising for tokamak operation at high wall temperature without excessive core contamination.

\section{Acknowledgements}

We acknowledge important discussions with R.W. Moir and D.K. Sze. Work was performed under the auspices of the U.S. Dept. of Energy by the University of California Lawrence Livermore National Laboratory under contract No. W-7405-Eng-48.

\section{References}

[1] ITER Physics Basis, in Nucl. Fusion 39 (1999), Chapt. 4, 2391.

[2] R.W. Moir, Nucl. Fusion 37 (1997) 557.

[3] M.A. Abdou, et al., Fusion Eng. Design 54 (2001) 181.

[4] R.F. Mattas, et al., Fusion Eng. Design 49-50 (2000) 127.

[5] T.D. Rognlien and M.E. Rensink, J. Nucl. Mater. 290-293 312 (2001).

[6] J.N. Brooks, T.D. Rognlien, D.N. Ruzic, and J.P. Allain, J. Nucl. Mater. 290-293 185 (2001).

[7] F. Najmabadi and the ARIES Team, Fusion Eng. Design 38 (1997) 3.

[8] T.D. Rognlien, P.N. Brown, R.B. Campbell, et al., Contr. Plasma Phys. 34 (1994) 362; G.R. Smith, P.N. Brown, R.B. Campbell, et al., J. Nucl. Mater. 220-222 (1995) 1024.

[9] F. Wising, D.A. Knoll, S. Krasheninnikov, and T.D. Rognlien, Contr. Plasma Phys., 36 (1996), 309.

[10] D.P. Stotler, D.E. Post, and D. Reiter, Bull. Am. Phys. Soc. 38 (1993) 1919.

[11] R.A. Hulse, Nucl. Tech./Fusion 3 (1983) 259.

[12] S.I. Braginskii, Transport processes in a plasma, Reviews of Plasma Physics; Vol. 1, Ed. M.A. Leontovich (Consultants Bureau, New York, 1965), p. 205.

Received September 6, 2001. 